Buchleser. Ribana Wollermann stellt die Frage, wie sich das Konzept der Corporate Social Responsibility sinnvoll auf Medienunternehmen übertragen lässt und vergleicht hierzu zehn aktuelle Veröffentlichungen aus der Kommunikations- und Wirtschaftswissenschaft. Caspar Schauseil fragt nach den strategischen Diversifizierungsmöglichkeiten von Zeitschriftenverlagen angesichts sinkender Werbeumsätze und stagnierender Leserzahlen. Mithilfe von Expertengesprächen werden neun Diversifizierungsmärkte identifiziert, die sich drei Grundstrategien (Content, Online, Offline) zuordnen lassen. Für diese potenziellen neuen Verlagsaktivitäten werden zwei Bewertungsprofile nach ressourcen- und marktorientierten Kriterien theoretisch abgeleitet. Beide Kriterienkataloge dienen der Strukturierung von Expertengesprächen, auf deren Basis das relative Potenzial der neun Diversifizierungsoptionen für Zeitschriftenverlage verglichen wird. Zur Auswertung der Gespräche wird auf die Methodiken der Nutzwertanalyse (ressourcenorientierte Kriterien) sowie des Analytical Hierarchy Process (marktbasierte Kriterien) zurückgegriffen. $J u$ dith Gentz beschäftigt sich schließlich mit dem Thema der Markenführung in Zeitschriftenverlagen, deren Bedeutung für den Unternehmenserfolg infolge der zunehmend cross-medialen Aktivitäten zunimmt. Das von ihr entwickelte Modell mit Gestaltungsparametern und Erfolgsfaktoren der Markenführung orientiert sich am sog. Open System View (OSV), der strategische Wettbewerbsvorteile auf eine optimale Ausschöpfung von unternehmensinternen Ressourcen und Ressourcenzuflüssen von außen zurückführt. Der Erfolg der Markenführung eines Unternehmens zeige sich im ökonomischen Markenwert. Seine Maximierung erfordere nach dem OSV eine optimale Interaktion mit der Unternehmensumwelt. Als zentralen qualitativen Erfolgsindikator sieht die $\mathrm{Au}$ torin das Markenvertrauen, das nach der Methodik des vernetzten Denkens mit anderen Indikatoren wie Markenzufriedenheit, Markenloyalität oder Markenimage zu einem Wirkungsnetz verbunden wird. Analytisch leitet sie hieraus zehn wesentliche Einflussgrößen auf den Erfolg der Markenführung ab.

$\mathrm{Zu}$ den acht wieder abgedruckten Beiträgen gehören zwei Aufsätze, die sich mit den Veränderungen im Journalismus als Folge des Internets beschäftigen: zum wachsenden Konflikt zwischen Aktualitätsdruck bzw. Bilderzwang und sorgfältiger Recherche (Michael Beuthner) sowie zum Verhältnis von professionellem Journalismus und User-generated Content (Stephan Weichert). Weitere Beiträge analysie- ren die Folgen der zunehmenden Zahl deutscher Kinofilmproduktionen auf deren wirtschaftlichen Erfolg (Oliver Castendyk); den Einfluss der Titelblattgestaltung von politischen Magazinen auf deren Einzelverkaufsabsatz, wobei Zeitreihenmodelle aus der Finanzmathematik verwendet werden (Marcel Garz $\mathcal{E}$ Armin Rott); die Erfahrungen mit der Einbeziehung von Externen in den Qualitätssicherungsprozess von öffentlich-rechtlichen Rundfunkanstalten (Anja Würzberg); die Wirkungen des Einsatzes von Fan-Pages als Marketing-Instrument für Zeitschriften (Lisa-Charlotte Walter). In einem weiteren Aufsatz wird der in Deutschland entwickelte dynamisch-transaktionale Ansatz (TDA) zur Medienwirkung mit empirischen Ergebnissen der Sozialpsychologie zusammengeführt und eine zentrale Aussage des TDA, wonach Medienstimuli im Rezeptionsprozess selbst eine veränderliche Größe sind, experimentell überprüft (Sabine Trepte, Leonhard Reinecke \& Katharina-Maria Behr). Die Herausgeberin selbst hat schließlich 2010 zusammen mit Mania Strube eine Wiederholungsstudie zu den Internationalisierungsstrategien der fünf größten deutschen Mediengruppen durchgeführt, deren Ergebnisse im aktuellen Sammelband erneut abgedruckt werden.

Auch wenn die meisten Beiträge des Sammelbandes bereits an anderer Stelle erschienen sind und er auch sicher nicht alle Kernthemen der medienwirtschaftlichen Forschung der letzten Dekade enthält, ist er aufgrund der Breite seiner Themenstellungen gut dazu geeignet, den interdisziplinären Blick seiner Leser zu schärfen. Kommunikationswissenschaftler erhalten einen guten Überblick über zentrale medienökonomische Fragestellungen, die sich aus dem aktuellen Wandlungsprozess des Mediensystems ergeben. Ebenso können sich Wirtschaftswissenschaftler, die sich mit Medienunternehmen oder Medienmärkten befassen, einen schnellen Überblick über zentrale Forschungsfelder der Kommunikationswissenschaft verschaffen.

Wolfgang Seufert

\section{Simon Sturm}

\section{Digitales Storytelling}

Eine Einführung in neue Formen des

Qualitätsjournalismus

Wiesbaden: Springer VS, 2013. - 169 S.

ISBN 978-3-658-02012-5

In seiner kalifornischen Ursprungsbedeutung meint der inzwischen auch hierzulande infla- 
tionär gebrauchte Begriff des „Digital Storytelling " genau das Gegenteil von professionellem Journalismus - nämlich eine Form von aktiver Medienarbeit: Einfache Menschen erzählen Aspekte ihres Alltagslebens in Form von kurzen digitalen Formaten. Jedermann soll lernen können, anderen zuzuhören, und zwar indem er ermuntert und befähigt wird, eigene Geschichten medial aufzubereiten und mit anderen zu teilen. Im Mittelpunkt steht dabei der Bildungswert von persönlich erzählten Geschichten - als reflexive Praxis, als pädagogische Strategie, als Mittel für Erziehungs- und Sozialarbeit, als Ermutigung zu politischem Engagement. Dabei ist es eher unwichtig, welche medialen Techniken dafür eingesetzt werden, nur einfach handhabbar und leicht zu erlernen sollten sie sein. Das „Center for Digital Storytelling“ beispielsweise (vgl.: http://storycenter.org/about-us/) betrachtet sein Kurskonzept als Werkzeug des Wandels in einer technisch und medial überladenen Welt. Hier geht es primär darum, dass möglichst viele Menschen zum kommunikativen Handeln befähigt werden. Die selbst entwickelte Geschichte kann dabei in einer großen Zahl von traditionellen und neuen medialen Techniken, also z. B. als Foto-, Audio- oder Videoreportage, als Comic, Podcast oder Game konzipiert und produziert werden, nicht nur in einem der neuen digitalen Social-Media-Formate. Ebenso wichtig wie die Geschichte selbst ist beim „Digital Storytelling“ übrigens die angstfreie Umgebung, in der sie produziert wird.

Simon Sturm hat sein „Digitales Storytelling“ als spezielle „Einführung in neue Formen des Qualitätsjournalismus“ konzipiert. Man kann darin ein spezifisches Informations- und Lernangebot für verunsicherte Journalisten sehen, die sich „im Sog der Digitalisierung“ befinden und ,an den altbewährten Darstellungsformen festklammern, die aus der analogen Welt stammen, noch bevor es Smartphones, Tablets, immer günstigere Daten-Flatrates ... gab“ (ebd., S. 4). Das Buch will angehenden und bereits im Beruf stehenden Journalisten „Mut machen und zeigen, dass es trotz aller Unkenrufe und wegbrechender Geschäftsmodelle auch viele Chancen gibt - Chancen für neue Formen des Qualitätsjournalismus"(Vorwort).

Mit der Idee von einem besseren, ambitionierteren „digitalen Qualitätsjournalismus“ knüpft Sturm zunächst an einschlägige Beiträge von Lilienthal, Niggemeier und Matzat an, die sich seit Längerem kritisch-analytisch (und weitgehend folgenlos) an der fehlenden Innovationsfreude der Verlage und der mangelnden Zahlungsbereitschaft des Publikums abgearbei- tet haben. Sodann schlägt Sturm einen eher optimistischen, praxisorientiert-pragmatischen Weg ein und nennt im Anschluss an Klaus Meier jene Qualitätsdimensionen, die auf praktisches Handeln in Redaktionen bezogen sind: redaktionelle Unabhängigkeit, originelle Recherche, relevante Aktualität, Dramaturgie und Emotionalität, Informationstiefe und Nutzerführung, technische Interaktivität und Nutzwert, Crossmedialität; diese sieben Dimensionen werden konsequent definiert und anschaulich erläutert.

Für seine „Grundlagen multimedialen Erzählens“, zur Erläuterung der Hoffnungen, die in neue Vermarktungsmodelle mittels TabletComputern gesetzt werden und zur Charakterisierung von Themen und Trends für neue Präsentationsmöglichkeiten hat Simon dann sehr heterogene Aspekte zusammengetragen: Definitionsversuche von Hypertext, Intraktivität und Storytelling, basale Kategorien der Dramaturgie, technische Merkmale von Tablet-Computern, die Geheimnisse der Touch-Navigation, einen Überblick über verschiedene Layoutund Navigationskonzepte bei journalistischen Tablet-Formaten, Game-Faktoren, Flow-Erleben. All dies und einiges mehr wird hier benannt und aufgelistet und durchweg richtig und plausibel beschrieben - jedoch leider kaum im Zusammenhang analysiert oder auf seine Relevanz hin untersucht.

Im Mittelpunkt der Darstellung steht sodann eine umfangreiche „Typologie digitaler Darstellungsformen", die Sturm zunächst medial differenziert und erst in einem zweiten Zugang über journalistisch-funktionsorientierte Formen beschreibt. In der medialen Differenzierung werden folgende Formen angesprochen: Schrifttext-Formen (Artikeltext, Teaser, FrageAntwort-Texte, Microblog, Eilmeldung, Nachrichtenticker und Kurztextgalerie), FotoFormen (digitales Foto, Bildergalerie/Slideshow, Audio-Slideshow, 360-Grad-Panorama/ 3D-Foto, Gigapan, Infinity-Foto und Zeitraffer-Foto), Audio-Formen (Audio-Clip, Audio-Stream, vertonter Artikeltext, Audio-Slideshow), Video-Formen (Video-Clip, Videoblog, Making-of-Video, Teaser-Video/Intro-Video, Videostream, 360-Grad-Video/3DVideo, Zeitraffer-Video, Multimedia-Reportage) und grafische Formen/Datenjournalismus (Infografik, Animation, interaktive Live-Karte, Daten-Mashup). In jeweils kurzen Abschnitten werden prägnante Merkmale genannt und Nutzungsmöglichkeiten anhand von konkreten Beispielen aus der aktuellen Online-Publizistik erläutert. Warum und wie lange es diese aufgeführten Möglichkeiten gibt, welche Tools auf 
welchen Geschäftsmodellen beruhen etc. wird dabei vernachlässigt, wie hier das Beispiel der inzwischen ja nicht mehr verfügbaren OnlineTools für „Vuvox-Collagen“ demonstriert.

Bei seinem ergänzenden, in den Erläuterungen noch deutlich kürzer ausfallenden funktionsorientierten Differenzierungsversuch - teilweise ist es nur eine Liste - berücksichtigt Sturm „Hypertext-Formen“ (kommentierte Linkliste, interaktive Zeitleiste, Tag-Cloud), „kommunikative Formen“ (Live-Chat, Schriftkommunikation zwischen Redaktion und Nutzern per E-Mail oder Kontaktformular, abonnierbare Newsletter, Kommunikation in Gästebüchern, Diskussionsforen, Weblogs, Newsgroups, Wikis, Microblogs etc., Kommunikation über Social Networks wie Facebook, Kommentar- und Bewertungsfunktionen, Leserfavoriten in Form von Top-Listen, Kommunikation über den Austausch von Daten, Leser-Kooperationen, z. B. kollaborative Recherche), „Echtzeit-Formen“ („Prozessjournalismus“: Liveticker, Liveblog, öffentliche Recherche) „spielerische Formen“ (Umfrage, Quiz/Wissens-Test, Newsgames/Simulation) sowie „aggregative/kurative Formen" (kurative NetzGeschichte, Themenpaket/Dossier, Multiperspektiven-Geschichte, kollaborative Geschichte).

Das alles ist möglich und das gibt es selbstverständlich auch in den journalistischen Online-Seiten hierzulande. Gewiss ist auch richtig, dass „gerade öffentlich-rechtliche Sender noch viel mehr aus ihrer Bewegtbild-Kompetenz machen und ganz neue Formen des digitalen Storytellings voranbringen “ (S. 144) könnten, auch dass ,jeder Journalist ein Grundverständnis dafür mitbringen sollte, wann welches Medium sinnvoll eingesetzt werden kann und wann nicht" (S. 145). Doch mit den sich aufdrängenden Anschlussfragen, was von dem Genannten wirklich relevant und zielführend ist, was $\mathrm{zu}$ welchen Kosten zu haben und mit welchem Nutzen für eine Stabilisierung des Interesses an journalistischen Angeboten eingesetzt werden könnte, beschäftigt sich das schmale Bändchen (146 Seiten Text plus Beispiel- und Quellenund Sachwort-Verzeichnisse) leider nicht.

Mich erinnert dieser im Definitorisch-Deskriptiven stecken gebliebene Überblick zum digitalen Storytelling in gewisser Weise an die Konzeption des Orbis sensualium pictus von Johann Amos Comenius (1592-1670), wo es in der „Invitatio“ im Dialog des Magisters (M.) mit dem Schüler (P.) heißt: „M. Veni, Puer! disce Sapere. P. Quid hoc est, Sapere? M. Omnia, qux necessaria, rectè intelligere, recte agere, rectè eloqui. P. Quis me hoc docebit? M. Ego, cum DEO. P. Quomodo? M. Ducam te, per omnia, ostendam tibi omnia, nominabo tibi omnia.“ ... Ich will Dich führen durch alle Dinge, ich will Dir zeigen alles, ich will Dir benennen alles ... Benennungen und Beschreibungen sind auch bei aktuellen Techniken sicher wichtig, können aber immer nur ein Anfang sein, denn für das Verständnis und die praktische Umsetzung sind recht Verstehen und recht Tun ebenfalls zwingend erforderlich. Im Übrigen waren die Dinge bei Comenius klüger ausgewählt und aufwändiger illustriert.

Wolfgang H. Swoboda

\section{Peter Szyszka (Hrsg.)}

Alles nur Theater?

Authentizität und Inszenierung in der Organisationskommunikation

Köln: Halem, 2012. - 304 S.

(Organisationskommunikation; 1)

\section{ISBN 978-3-86962-044-2}

Die Beiträge des vorliegenden Bandes sind das Ergebnis einer gleichnamigen Tagung der Fachgruppe Public Relations und Organisationskommunikation. Der Herausgeber präsentiert damit zugleich den ersten Band einer neuen Reihe im Herbert van Halem Verlag zur Organisationskommunikation. Die Programmatik dieser neuen Reihe ist gleichermaßen anspruchsvoll wie dringlich, geht es doch darum, die Unschärfe vieler Begriffe in der PR und Organisationskommunikation durch eine theoriegeleitete Forschung zu beseitigen, einschließlich des Begriffs der Organisationskommunikation selbst. Authentizität, in der Alltagssprache häufig in einem Atemzug mit Glaubwürdigkeit genannt, erweist sich als eine geeignete (Test-)Kandidatin für ein derartiges Vorhaben.

Der Band enthält 12 Einzelbeiträge, gerahmt durch die Ausführungen zur Programmatik der neuen Reihe durch den Herausgeber und einen Vortrag zum Forschungsfeld der Organisationskommunikation, den Ulrich Saxer am 15.6.2010 in Wien gehalten hat. Diese Ausführungen bilden sozusagen die erste, weitere Klammer, die sich auf das Verständnis der Organisationskommunikation bezieht. Der Herausgeber plädiert für ein (sehr) weites Begriffsverständnis (Kommunikation in, von und über Organisationen) und Ulrich Saxer beschreibt den beschwerlichen Weg, dieses Forschungsfeld als Gegenstand der Publizistik- und Kommunikationswissenschaft zu etablieren. Daneben existiert eine thematisch enger gefasste Klammer, die sich nicht nur auf die theoretische 